\title{
Minimal Parallelism and Number of Membrane Polarizations ${ }^{\star}$
}

\author{
Artiom Alhazov ${ }^{1}$ \\ Institute of Mathematics and Computer Science \\ Academy of Sciences of Moldova \\ Chişinau, MD-2028, Moldova \\ E-mail: artiom@math.md
}

Summary. It is known that the satisfiability problem (SAT) can be efficiently solved by a uniform family of $P$ systems with active membranes that have two polarizations working in a maximally parallel way. We study P systems with active membranes without non-elementary membrane division, working in minimally parallel way. The main question we address is what number of polarizations is sufficient for an efficient computation depending on the types of rules used.

In particular, we show that it is enough to have four polarizations, sequential evolution rules changing polarizations, polarizationless non-elementary membrane division rules and polarizationless rules of sending an object out. The same problem is solved with the standard evolution rules, rules of sending an object out and polarizationless non-elementary membrane division rules, with six polarizations. It is an open question whether these numbers are optimal.

* The author acknowledges project 4032 from the Science and Technology Center in Ukraine and project TIC2003-09319-C03-01 from the Rovira i Virgili University. The preliminary version of this paper was published in the pre-proceedings of the Workshop in Membrane Computing, Leiden, 2006 and presented at the seminar of the Research Group on Mathematical Linguistics, Tarragona. 


\section{Introduction}

Membrane computing with symbol-objects is a biologically inspired framework of distributed parallel multiset processing; see [10] for an overwiew and [13] for the comprehensive bibliography. The most addressed questions are completeness (solving every solvable problem) and efficiency (solving hard problems in a feasible time). We focus on the latter.

An interesting class of membrane systems are those with active membranes (see [9]), where membrane division can be used for solving computationally hard problems in polynomial time. Let us mention a few results:

- A semi-uniform solution to SAT using three polarizations and division for non-elementary membranes, [9].

- A polarizationless solution, [2].

- Only division for elementary membranes, with three polarizations [11].

- A uniform solution, with elementary membrane division, [12].

- Only two polarizations, in a uniform way, with elementary membrane division [3].

- Computational completeness of P systems with three polarizations and three membranes [10].

- Only two polarizations and two membranes [6].

- Only one membrane, with two polarizations [4, 5].

- Polarizationless systems are complete, with no known bound on the number of membranes [1].

- Solving SAT in a minimally parallel way, using non-elementary membrane division (replicating both objects and inner membranes) [7].

- Polarizations avoided by using rules that change membrane labels. To the best of the author's knowledge the rules are either cooperative or nonelementary division as above [8].

Given a P system, a rule and an object, whether the rule is applicable to the object in some membrane might depend on both the membrane label (that usually cannot be changed) and the membrane polarization. Essentially, the number of polarizations is the number of states that can be encoded directly on the membrane.

Minimal parallelism provides less synchronization between the objects, so one might expect the need for stronger control (i.e., more polarizations). It is not difficult to construct the system so that the rules are global (i.e., the membrane labels are not distinguished), probably by not adding additional 
polarizations. In this way, the results dealing with the number of polarizations can be reformulated in terms of number of membrane labels (in which case the systems have no polarizations, but the rules are allowed to modify membrane labels).

\section{Preliminaries}

\subsection{Solvability by $P$ systems with input}

Definition 1. A P system with input is a tuple $\left(\Pi, \Sigma, i_{\Pi}\right)$, where (a) $\Pi$ is a $P$ system with working alphabet, with $m$ membranes labelled with $1, \cdots, m$, and initial multisets $w_{1}, \cdots, w_{m}$ (over $O-\Sigma$ ) associated with them; $(b) \Sigma \subseteq O$ is an (input) alphabet, (c) $i_{\Pi}$ is the label of a distinguished (input) membrane.

The initial configuration of $\left(\Pi, \Sigma, i_{\Pi}\right)$ with an input multiset $w$ over $\Sigma$ is

$$
\left(\mu, w_{1}, \cdots, w_{i_{\Pi}} \cup w, \cdots, w_{m}\right) .
$$

We call $\left(\Pi, \Sigma, i_{\Pi}\right)$ a decisional $\mathrm{P}$ system with input if there exist two distinguished objects yes, no $\in O$ and for any valid input (see cod function in the definition below) all its computations send to the environment exactly one object: either yes (in this case the computation is called an accepting one) or no. Moreover, $\left(\Pi, \Sigma, i_{\Pi}\right)$ is called confluent if for any valid input all its computations halt in the same configuration.

Definition 2. Consider a decision problem $X=\left(I_{X}, \theta_{X}\right): I_{X}$ is the set of possible instances of $X$ and $\theta_{X}$ is a Boolean function over $I_{X}$. We say that $X$ is solvable in polynomial time by a uniform family of $P$ systems $\Pi=(\Pi(n))_{n \in \mathbb{N}}$ if the following conditions hold:

- The family $\Pi$ is polynomially constructible: i.e., there exists a deterministic Turing machine constructing the system $\Pi(n)$ from $n$ in polynomial time.

- There exists a pair (s,cod) of polynomial-time computable functions mapping every instance $u \in I_{X}$ of the problem $X$ into a natural number and a multiset (over the alphabet of $\Pi(s(u))$ ), respectively. The instance $u$ is to be solved by a system $\Pi(s(u))$ with the multiset cod $(u)$ placed in the input membrane, as described below.

- The family $\Pi$ is polynomially bounded with respect to $(X, \operatorname{cod}$,s): i.e., there exists a polynomial function $p(n)$ such that for each $u \in I_{X}$ every computation of the system $\Pi(s(u))$ with input cod $(u)$ halts in at most $p(s(u))$ steps. 
- The family $\Pi$ is sound with respect to $\left(X, \operatorname{cod}\right.$, s): i.e., for each $u \in I_{X}$ if there exists an accepting computation of $\Pi(s(u))$ with input $\operatorname{cod}(u)$, then $\theta_{X}(u)=$ 1.

- The family $\Pi$ is complete with respect to $\left(X, \operatorname{cod}\right.$, s): i.e., for each $u \in I_{X}$ if $\theta_{X}(u)=1$, then every computation of $\Pi(s(u))$ with input $\operatorname{cod}(u)$ is an accepting one.

\subsection{P systems with active membranes}

Definition 3. A P system with active membranes is a P system with the working alphabet $O$, the set $H$ of membrane labels, the set $E$ of polarizations, and the rules of the following forms:

(a) $\left.[a]_{a} u\right]_{h}^{e}$ for $a \in O, u \in O^{*}, h \in H$ and $e \in E$. These are object evolution rules. An object $a \in O$ in the region associated with a membrane with label $h$ and polarization e evolves to a multiset $u \in O^{*}$.

(b) $a[]_{h}^{e} \rightarrow[b]_{h}^{e^{\prime}}$ for $a, b \in O, h \in H$ and $e, e^{\prime} \in E$. These are send-in communication rules. An object a from the region immediately outside a membrane with label $h$ and polarization $e$ is introduced in to this membrane, transformed into $b$ and the polarization of the membrane is changed to $e^{\prime}$.

(c) $[a]_{h}^{e} \rightarrow[]_{h}^{e^{\prime}} b$ for $a, b \in O, h \in H$ and $e, e^{\prime} \in E$. These are send-out communication rules. An object $a$ is sent out from the region associated with a membrane with label $h$ and polarization e to the region immediately outside, transformed into $b$ and the polarization of the membrane is changed to $e^{\prime}$.

(d) $[a]_{h}^{e} \rightarrow a$ for $a, b \in O, h \in H$ and $e \in E$. These are dissolution rules. A membrane with label $h$ and polarization $e$ is dissolved in reaction with an object $a$, and transformed into $b$. The skin is never dissolved.

(e) $[a]_{h}^{e} \rightarrow[b]_{h}^{e^{\prime}}[c]_{h}^{e^{\prime \prime}}$ for $a, b, c \in O, h \in H$ and $e, e^{\prime}, e^{\prime \prime} \in E$. These are division rules for elementary membranes. An elementary membrane can be divided into two membranes with the same label, possibly with different polarizations, possibly transforming some objects.

Generally, rules of type $(a)$ are executed in parallel, while at most one rule out of all rules of types $(b),(c),(d),(e)$ can be applied to the same membrane in the same step. We will also speak about the sequential version

$$
\left(a_{s}^{\prime \prime}\right)[a]_{h}^{e} \rightarrow[u]_{h}^{e^{\prime}} \text { for } a \in O, u \in O^{*}, h \in H \text { and } e, e^{\prime} \in E .
$$

of rules $(a)$ (let us use " to indicate that the rule is allowed to change the polarization of the membrane) and their modifications $\left(b_{0}\right),\left(c_{0}\right),\left(d_{0}\right),\left(e_{0}\right)$, 
$\left(a_{0 s}^{\prime}\right),\left(b_{0}^{\prime}\right),\left(c_{0}^{\prime}\right),\left(e_{0}^{\prime}\right)$ (here, 0 represents that the rules neither distinguish polarization nor change it, while ' means that the rule is allowed to change membrane label).

\subsection{Minimal parallelism}

These rules are applied according to the following principles:

- The rules of type (a) may be applied in parallel. In one step, a membrane can be the subject of only one rule of types $\left(a_{0 s}^{\prime}\right),\left(a_{s}^{\prime \prime}\right)$ and $(b),(c),(d),(e)$ with their modifications.

- In one step, one object of a membrane can be used by only one rule (nondeterministically chosen), but for every membrane at least one object that can evolve by one rule of any form, must evolve (no rules associated to a membrane are applied only if none are applicable).

- If at the same time a membrane is divided by a rule of type $(e)$ and there are objects in this membrane which evolve by means of rules of type $(a)$, then we assume that the evolution rules of type $(a)$ are used first, and then the division is produced. Of course, this process takes only one step.

\section{Using rules $\left(a_{s}^{\prime \prime}\right)$}

The three size parameters of the SAT problem are the number $m$ of clauses, the number $n$ of variables and the total number $l$ of occurrences of variables in clauses (clearly, $l \leq m n$ : without restricting generality, we could assume that no variable appears in the same clause more than once, with or without negation).

Theorem 1. A uniform family of confluent $P$ systems with rules $\left(a_{s}^{\prime \prime}\right),\left(c_{0}\right),\left(e_{0}\right)$ working in minimally parallel way can solve SAT with four polarizations in $O(l(m+n))$ number of steps.

Proof. The main idea of the construction is to implement a maximally parallel step sequentially. To do so, a "control" object will change the polarization, and then an input object or a clause object will restore it. Since the input is encoded in $l$ objects, changing and restoring polarization will happen $l$ times, and the counting is done by the "control" object.

Let us consider a propositional formula in the conjunctive normal form:

TRIANGLE 6 • December 2011 


$$
\begin{aligned}
\beta & =C_{1} \vee \cdots \vee C_{m}, \\
C_{i} & =y_{i, 1} \wedge \cdots \wedge y_{i, l_{i}, 1 \leq i \leq m, \text { where }} \\
y_{i, k} & \in\left\{x_{j}, \neg x_{j} \mid 1 \leq j \leq n\right\}, 1 \leq i \leq m, 1 \leq k \leq l_{i}, \\
l & =\sum_{i=1}^{m} l_{i} .
\end{aligned}
$$

Let us encode the instance of $\beta$ in the alphabet $\Sigma(\langle n, m, l\rangle)$ by multisets $X, X^{\prime}$ of the clause-variable pairs such that the variable appears in the clause without negation, with negation or neither:

$$
\begin{aligned}
\Sigma(\langle n, m, l\rangle)= & \left\{v_{j, i, 1, s} \mid 1 \leq j \leq m, 1 \leq i \leq n, 1 \leq s \leq 2\right\}, \\
X= & \left\{\left(v_{j, i, 1,1}, 1\right) \mid x_{i} \in\left\{y_{j, k} \mid 1 \leq k \leq l_{j}\right\},\right. \\
& 1 \leq j \leq m, 1 \leq i \leq n\}, \\
X^{\prime}= & \left\{\left(v_{j, i, 1,2}, 1\right) \mid \neg x_{i} \in\left\{y_{j, k} \mid 1 \leq k \leq l_{j}\right\},\right. \\
& 1 \leq j \leq m, 1 \leq i \leq n\} .
\end{aligned}
$$

We construct the following P system:

$$
\begin{aligned}
\Pi(\langle n, m, l\rangle)= & \left(O, H, E,\left[[]_{2}^{0}[]_{3}^{0}\right]_{1}^{0}, w_{1}, w_{2}, w_{3}, R\right), \text { with } \\
O= & \left\{v_{j, i, k, s} \mid 1 \leq j \leq m, 1 \leq i \leq n,\right. \\
& 1 \leq k \leq m+n+1,1 \leq s \leq 4\} \\
& \cup\left\{d_{i, k} \mid 1 \leq i \leq m+n+1,1 \leq k \leq 2 l\right\} \\
& \cup\left\{t_{i, k}, f_{i, k} \mid 1 \leq i \leq n, 1 \leq k \leq l\right\} \\
& \cup\left\{d_{i} \mid 1 \leq i \leq m+n+1\right\} \cup\{S, Z, \text { yes, no }\} \\
& \cup\left\{z_{k} \mid 1 \leq k \leq(4 l+3) n+m(4 l+1)+2\right\} \\
w_{1}= & \lambda, w_{2}=d_{1}, w_{3}=z_{0}, H=\{1,2,3\}, E=\{0,1,2,3\},
\end{aligned}
$$

and the rules are listed below. The computation consists of three stages.

1. Producing $2^{n}$ membranes with label 2 , corresponding to the possible assignments of variables $x_{1}, \cdots, x_{n}$ and selecting clauses that are satisfied for every assignment (groups $\mathrm{A}$ and $\mathrm{C}$ of rules).

2. Checking whether all clauses are satisfied for all assignments (groups of rules $\mathrm{B}$ and $\mathrm{D})$.

3. Generating yes from the positive answer, and sending it to the environment. Generating no from the timeout (during the first two stages the number of steps is counted in the object in membrane with label 3) and 
sending it to the environment if there is no positive answer (groups of rules $\mathrm{E}$ and $\mathrm{F}$ ).

Stage 1 consists of $n$ cycles and stage 2 consists of $m$ cycles. Each cycle's aim is to process all $l$ objects: i.e., each object counts the number of cycles completed, and in the first stage the clauses are evaluated while in the second stage the presence of each clause is checked.

In the case of maximal parallelism, a cycle can be performed in a constant number of (actually, one or two) steps, while minimal parallelism cannot guarantee that all objects are processed. The solution used here is the following. A cycle consists of marking (setting the last index to 3 or 4 ) all $l$ objects one by one while performing the necessary operation, and then unmarking (setting the last index to 1 or 2) all of them. Marking or unmarking an object happens in two steps: the control object changes the polarization from 0 to 1, 2 (to mark) or 3 (to unmark), and then one of the objects that has not yet been (un)marked is processed, resetting the polarization to 0 .

\section{Control objects in membrane 2: select clauses}

A1 (for variable $i$ : divide)

$$
\left[d_{i}\right] \rightarrow\left[t_{i, 0}\right]\left[f_{i, 0}\right], 1 \leq i \leq n
$$

A2 (process and mark all $l$ objects)

$$
\begin{aligned}
& {\left[t_{i, k-1}\right]^{0} \rightarrow\left[t_{i, k}\right]^{1}, 1 \leq i \leq n, 1 \leq k \leq l} \\
& {\left[f_{i, k-1}\right]^{0} \rightarrow\left[f_{i, k}\right]^{2}, 1 \leq i \leq n, 1 \leq k \leq l}
\end{aligned}
$$

A3 (prepare to unmark objects)

$$
\begin{aligned}
& {\left[t_{i, l}\right]^{0} \rightarrow\left[d_{i, 0}\right]^{0}, 1 \leq i \leq n} \\
& {\left[f_{i, l}\right]^{0} \rightarrow\left[d_{i, 0}\right]^{0}, 1 \leq i \leq n}
\end{aligned}
$$

A4 (unmark all $l$ objects)

$$
\left[d_{i, k-1}\right]^{0} \rightarrow\left[d_{i, k}\right]^{3}, 1 \leq i \leq n, 1 \leq k \leq l
$$

A5 (switch to the next variable)

$$
\left[d_{i, l}\right]^{0} \rightarrow\left[d_{i+1}\right]^{0}, 1 \leq i \leq n
$$

\section{Control objects in membrane 2: check clauses}

B1 (test if clause $i$ is satisfied)

$$
\left[d_{n+i}\right]^{0} \rightarrow\left[d_{n+i, 1}\right]^{2}, 1 \leq i \leq m
$$

B2 (process and mark the other $l-1$ objects)

$$
\left[d_{n+i, k-1}\right]^{0} \rightarrow\left[d_{n+i, k}\right]^{1}, 1 \leq i \leq m, 1 \leq k \leq l
$$

B3 (unmark all $l$ objects)

$$
\left[d_{n+i, l+k-1}\right]^{0} \rightarrow\left[d_{n+i, l+k}\right]^{3}, 1 \leq i \leq m, 1 \leq k \leq l
$$


B4 (switch to the next clause)

$\left[d_{n+i, 2 l}\right]^{0} \rightarrow\left[d_{n+i+1}\right]^{0}, 1 \leq i \leq m$

B5 (send a positive answer)

$\left[d_{m+n+1}\right] \rightarrow[] S$

\section{Input objects in membrane 2: select clauses}

C1 (mark an object)

$\left[v_{j, i, k, s}\right]^{p} \rightarrow\left[v_{j, i, k+1, s+2}\right]^{0}$,

$1 \leq i \leq m, 1 \leq j \leq n, 1 \leq k \leq m, k \neq m, 1 \leq s \leq 2,1 \leq p \leq 2$

C2 (a true variable present without negation or a false variable present with negation satisfies the clause)

$\left[v_{j, i, i, s}\right]^{s} \rightarrow\left[v_{j, i, i+1,3}\right]^{0}, 1 \leq i \leq m, 1 \leq j \leq n, 1 \leq s \leq 2$

C3 (a true variable present with negation or a false variable present without negation does not satisfy the clause)

$\left[v_{j, i, i, 3-s}\right]^{s} \rightarrow\left[v_{j, i, i+1,4}\right]^{0}, 1 \leq i \leq m, 1 \leq j \leq n, 1 \leq s \leq 2$

C4 (unmark an object)

$\left[v_{j, i, k, s+2}\right]^{3} \rightarrow\left[v_{j, i, k, s}\right]^{0}$,

$1 \leq i \leq m, 1 \leq j \leq n, 2 \leq k \leq m+1,1 \leq s \leq 2$

Input objects in membrane 2: check clauses

D1 (check if the clause is satisfied by at least one variable)

$\left[v_{j, i, m+j, 1}\right]^{2} \rightarrow\left[v_{j, i, k+1,3}\right]^{0}, 1 \leq i \leq m, 1 \leq j \leq n, 1 \leq s \leq 2$

D2 (mark an object)

$\left[v_{j, i, m+k, s}\right]^{1} \rightarrow\left[v_{j, i, k+1, s+2}\right]^{0}$,

$1 \leq i \leq m, 1 \leq j \leq n, 1 \leq k \leq n, 1 \leq s \leq 2$

D3 (unmark an object)

$\left[v_{j, i, m+k, s+2}\right]^{3} \rightarrow\left[v_{j, i, k, s}\right]^{0}$,

$1 \leq i \leq m, 1 \leq j \leq n, 2 \leq k \leq n+1,1 \leq s \leq 2$

\section{Control objects in membrane 3}

E1 (count)

$$
\left[z_{k-1}\right]^{0} \rightarrow\left[z_{k}\right]^{0}, 1 \leq k \leq N=(4 l+3) n+m(4 l+1)+2
$$

E2 (send time-out object)

$\left[z_{N}\right] \rightarrow[] Z$

\section{Control objects in the skin membrane}

F1 (a positive result generates the answer)

$[S]^{0} \rightarrow[\text { yes }]^{1}$ 
F2 (without the positive answer, the time-out generates the negative answer) $[\mathrm{Z}]^{0} \rightarrow[\mathrm{no}]^{0}$

F3 (send the answer)

$$
\begin{aligned}
& {[\text { yes }] \rightarrow[\text { ] yes }} \\
& {[\text { no }] \rightarrow[] \text { no }}
\end{aligned}
$$

Let us now explain how the system works in greater detail.

Like the input objects, the control objects keep track of the number of cycles completed. The control object also remembers whether marking or unmarking takes place, as well as the number of objects already (un)marked. Moreover, the control object is responsible for sending the "right" information to the objects via polarization: in stage 1,1 if the variable is true, and 2 if the variable is false; in stage 2, 1 if the clause has already been found, and 2 if the clause is being checked for.

During the first stage, an object $v_{j, i, 1, s}$ is transformed into $v_{j, i, n+1, t}$, where $t=1$ if variable $x_{j}$ satisfies clause $C_{i}$, and $t=2$ if not. The last index changes from $s$ to $t$ when the third index is equal to $i$. Notice that, although only information about what clauses are satisfied seems to be necessary for checking if $\beta$ is true for the given assignment of the variables, such information as the number of cycles completed is kept for synchronization purposes, and the other objects are kept so that their total number remains $l$. The control object $d_{1}$ is transformed into $d_{n+1}$. Stage 1 takes $(4 l+3) n$ steps.

If some clause is not satisfied, then the computation in the corresponding membrane is "stuck" with polarization 2. Otherwise, during the second stage an object $v_{j, i, n+1, t}$ is transformed into $v_{j, i, n+m+1, t}$, while the control object $d_{n+1}$ becomes $d_{m+n+1}$. Stage 2 takes $m(4 l+1)$ steps, plus one extra step to send objects $S$ to skin, if any.

After stage 2 is completed, one copy of $S$, if any, is transformed into yes, changing the polarization of the skin membrane. If a yes is sent out, at the same time object $Z$ comes to the skin from region 3. If the polarization of the skin is still $0, Z$ changes to no, and is then sent out. Depending on the answer, stage 3 takes 2 or 4 steps. In either case, the result is sent out in the last step of the computation.

Notice that membrane labels are not indicated in the rules. This means that the system is organized in such a way that the rules are global: i.e., the system would work equally well if it started with the configuration $\mu=\left[w_{1}\left[w_{2}\right]_{1}^{0}\left[w_{3}\right]_{1}^{0}\right]_{1}^{0}$. The labels are only given for the simplicity of explanation. 
Using the remark at the end of the Introduction, we can obtain the following

Corollary 1. A uniform family of confluent polarizationless $P$ systems with rules $\left(a_{0 s}^{\prime}\right),\left(c_{0}\right),\left(e_{0}\right)$ working in minimally parallel way can solve SAT with membrane labels of four kinds.

The statement follows directly from the possibility of rewriting a global rule $[a]^{e} \rightarrow[u]^{e^{\prime}}$ of type $\left(a_{s}^{\prime \prime}\right)$ as a rule $[a]_{e} \rightarrow[u]_{e^{\prime}}$ of type $\left(a_{0 s}^{\prime}\right)$ (which is polarizationless but can change the membrane label).

\section{Using rules $(a)$}

An informal idea in this section is to replace rules of type $\left(a_{s}^{\prime \prime}\right)$ with rules (a) producing additional objects, and rules $(c)$, sending an additional object out to change the polarization.

Theorem 2. A uniform family of confluent $P$ systems with rules $(a),(c),\left(e_{0}\right)$ working in minimally parallel way can solve SAT with six polarizations in $O(l(m+$ n)) number of steps.

Proof. The strategy used in the construction below is similar to that of the previous theorem. However, since the application of the evolution rules no longer changes the polarization of the membrane, the control symbols $d_{i, k}$, $t_{i, k}, f_{i, k}$ no longer "operate" in polarization 0 , but rather in a polarization that toggles between 0 (for even $k$ ) and 5 (for odd $k$ ), to prevent multiple applications of evolution rules in one row in the same membrane. Moreover, the input objects are actually allowed to evolve in parallel (and the degree of parallelism is chosen non-deterministically), but at the end of both halves of a cycle the number of extra objects produced can be counted, to make sure that all $l$ objects have been processed.

For the same propositional formula

$$
\begin{aligned}
\beta & =C_{1} \vee \cdots \vee C_{m} \\
C_{i} & =y_{i, 1} \wedge \cdots \wedge y_{i, l_{i}}, 1 \leq i \leq m, \text { where } \\
y_{i, k} & \in\left\{x_{j}, \neg x_{j} \mid 1 \leq j \leq n\right\}, 1 \leq i \leq m, 1 \leq k \leq l_{i} \\
l & =\sum_{i=1}^{m} l_{i} .
\end{aligned}
$$


and the same encoding of the instance of $\beta$ in the alphabet $\Sigma(\langle n, m, l\rangle)$ by multisets $X, X^{\prime}$,

$$
\begin{aligned}
\Sigma(\langle n, m, l\rangle)= & \left\{v_{j, i, 1, s} \mid 1 \leq j \leq m, 1 \leq i \leq n, 1 \leq s \leq 2\right\}, \\
X= & \left\{\left(v_{j, i, 1,1}, 1\right) \mid x_{i} \in\left\{y_{j, k} \mid 1 \leq k \leq l_{j}\right\},\right. \\
& 1 \leq j \leq m, 1 \leq i \leq n\}, \\
X^{\prime}= & \left\{\left(v_{j, i, 1,2}, 1\right) \mid \neg x_{i} \in\left\{y_{j, k} \mid 1 \leq k \leq l_{j}\right\},\right. \\
& 1 \leq j \leq m, 1 \leq i \leq n\} .
\end{aligned}
$$

we construct the following P system:

$$
\begin{aligned}
\Pi(\langle n, m, l\rangle)= & \left(O, H, E,\left[[]_{2}^{0}[]_{3}^{0}\right]_{1}^{0}, w_{1}, w_{2}, w_{3}, R\right), \text { with } \\
O= & \left\{v_{j, i, k, s} \mid 1 \leq j \leq m, 1 \leq i \leq n,\right. \\
& 1 \leq k \leq m+n+1,1 \leq s \leq 4\} \\
\cup & \left\{d_{i, k} \mid 1 \leq i \leq m+n+1,1 \leq k \leq 2 l\right\} \\
& \cup\left\{t_{i, k}, f_{i, k} \mid 1 \leq i \leq n, 1 \leq k \leq l\right\} \\
& \cup\left\{d_{i} \mid 1 \leq i \leq m+n+1\right\} \cup\{S, Z, \text { yes, no }\} \\
& \cup\left\{z_{k} \mid 1 \leq k \leq(4 l+3) n+m(4 l+1)+2\right\} \\
& \cup\left\{o_{i, j} \mid 0 \leq i \leq 5,0 \leq j \leq 5\right\} \\
w_{1}= & \lambda, w_{2}=d_{1}, w_{3}=z_{0}, H=\{1,2,3\}, E=\{0,1,2,3,4,5\},
\end{aligned}
$$

and the rules are listed below. The computation stages are the same as in the previous proof.

1. Producing $2^{n}$ membranes for all the possible assignments of variables; selecting satisfied clauses (groups A and C).

2. Checking whether all clauses are satisfied (groups B and D).

3. Generating the answer and sending it to the environment (groups $\mathrm{E}$ and F).

Stage 1 consists of $n$ cycles and stage 2 consists of $m$ cycles. Each cycle's aim is to process all $l$ objects: i.e., each object counts the number of cycles completed. In the first stage the clauses are evaluated while in the second stage the presence of each clause is checked.

A cycle consists of marking (setting the last index to 3 or 4 ) all $l$ objects one by one while performing the necessary operation, and then unmarking (setting the last index to 1 or 2) them. An object is generally marked or unmarked in five steps: 
1. The control object produces two "polarization changers".

2. One of them changes the polarization from 0 or 5 to 1,2 (to mark) or 3 (to unmark).

3. One of the objects that has not yet been (un)marked is processed, producing a "witness" - yet another "polarization changer".

4. The "witness" switches the polarization to 4 .

5. The second "changer" produced in step 1 of this routine changes the polarization to 5 or 0 .

Notice, however, that "step" 3 might actually take more than one step (more objects can be (un)marked in parallel, or even in a row, thus creating a supply of "witnesses"). Step 4 might actually be executed in parallel with the last step of "step" 3 (sending out a previous "witness" while producing more). Finally, "step" 3 might even be skipped if a previous "witness" is already there. What matters is that the whole (un)marking routine should take at most $5 l$ steps.

\section{Changing the polarization of membrane 2}

O1 (change from $i$ to $j$ )

$$
\left[o_{i, j}\right]^{i} \rightarrow[]^{j} o_{4,5}, 0 \leq i \leq 5,0 \leq j \leq 5
$$

O2 ("witnesses" of D2 are "compatible" with "witnesses" of D1; this does not interfere with the rest of the computation)

$$
\left[o_{1,4}\right]^{2} \rightarrow[]^{4} o_{4,5}
$$

\section{Control objects in membrane 2: select clauses}

A1 (for variable $i$ : divide)

$$
\left[d_{i}\right] \rightarrow\left[t_{i, 0}\right]\left[f_{i, 0}\right], 1 \leq i \leq n
$$

A2 (process and mark all $l$ objects)

$$
\begin{aligned}
& {\left[t_{i, k-1} \rightarrow t_{i, k} o_{0,1} o_{4,5}\right]^{0}, 1 \leq i \leq n, 1 \leq k \leq l, k \text { is odd }} \\
& {\left[f_{i, k-1} \rightarrow f_{i, k} o_{0,2} o_{4,5}\right]^{0}, 1 \leq i \leq n, 1 \leq k \leq l, k \text { is odd }} \\
& {\left[t_{i, k-1} \rightarrow t_{i, k} o_{5,1} o_{4,0}\right]^{5}, 1 \leq i \leq n, 1 \leq k \leq l, k \text { is even }} \\
& {\left[f_{i, k-1} \rightarrow f_{i, k} o_{5,2} o_{4,0}\right]^{5}, 1 \leq i \leq n, 1 \leq k \leq l, k \text { is even }}
\end{aligned}
$$

A3 (prepare to unmark objects)

$$
\begin{aligned}
& {\left[t_{i, l} \rightarrow d_{i, 0}\right]^{0}, 1 \leq i \leq n, \text { if } l \text { is even }} \\
& {\left[f_{i, l} \rightarrow d_{i, 0}\right]^{0}, 1 \leq i \leq n, \text { if } l \text { is even }} \\
& {\left[t_{i, l} \rightarrow d_{i, 0} 0_{5,0}\right]^{5}, 1 \leq i \leq n, \text { if } l \text { is odd }} \\
& {\left[f_{i, l} \rightarrow d_{i, 0} o_{5,0}\right]^{5}, 1 \leq i \leq n, \text { if } l \text { is odd }}
\end{aligned}
$$


A4 (unmark all $l$ objects)

$\left[d_{i, k-1} \rightarrow d_{i, k} o_{0,3} o_{4,5}\right]^{0}, 1 \leq i \leq n, 1 \leq k \leq l, k$ is odd

$\left[d_{i, k-1} \rightarrow d_{i, k} 0_{5,3} 0_{4,0}\right]^{0}, 1 \leq i \leq n, 1 \leq k \leq l, k$ is even

A5 (switch to the next variable)

$\left[d_{i, l} \rightarrow d_{i+1}\right]^{0}, 1 \leq i \leq n$, if $l$ is even

$\left[d_{i, l} \rightarrow d_{i+1} o_{5,0}\right]^{5}, 1 \leq i \leq n$, if $l$ is odd

\section{Control objects in membrane 2: check clauses}

B1 (test if clause $i$ is satisfied)

$\left[d_{n+i} \rightarrow d_{n+i, 1} o_{0,2} o_{4,5}\right]^{0}, 1 \leq i \leq m$

B2 (process and mark the other $l-1$ objects)

$\left[d_{n+i, k-1} \rightarrow d_{n+i, k} o_{0,1} o_{4,5}\right]^{0}, 1 \leq i \leq m, 1 \leq k \leq l, k$ is odd

$\left[d_{n+i, k-1} \rightarrow d_{n+i, k} o_{5,1} o_{4,0}\right]^{0}, 1 \leq i \leq m, 1 \leq k \leq l, k$ is even

B3 (unmark all $l$ objects)

$\left[d_{n+i, l+k-1} \rightarrow d_{n+i, l+k} o_{0,3} o_{4,5}\right]^{0}, 1 \leq i \leq m, 1 \leq k \leq l, l+k$ is odd

$\left[d_{n+i, l+k-1} \rightarrow d_{n+i, l+k} O_{5,3} O_{4,0}\right]^{0}, 1 \leq i \leq m, 1 \leq k \leq l, l+k$ is odd

B4 (switch to the next clause)

$\left[d_{n+i, 2 l} \rightarrow d_{n+i+1}\right]^{0}, 1 \leq i \leq m$

B5 (send a positive answer)

$\left[d_{m+n+1}\right]^{0} \rightarrow[]^{0} S$

\section{Input objects in membrane 2: select clauses}

C1 (mark an object)

$\left[v_{j, i, k, s} \rightarrow v_{j, i, k+1, s+2} o_{p, 4}\right]^{p}$,

$1 \leq i \leq m, 1 \leq j \leq n, 1 \leq k \leq m, k \neq m, 1 \leq s \leq 2,1 \leq p \leq 2$

C2 (a true variable present without negation or a false variable present with negation satisfies the clause)

$\left[v_{j, i, i, s} \rightarrow v_{j, i, i+1,3} o_{s, 4}\right]^{s}, 1 \leq i \leq m, 1 \leq j \leq n, 1 \leq s \leq 2$

C3 (a true variable present with negation or a false variable present without negation does not satisfy the clause)

$\left[v_{j, i, i, 3-s} \rightarrow v_{j, i, i+1,4} o_{s, 4}\right]^{s}, 1 \leq i \leq m, 1 \leq j \leq n, 1 \leq s \leq 2$

C4 (unmark an object)

$\left[v_{j, i, k, s+2} \rightarrow v_{j, i, k, s} o_{3,4}\right]^{3}$,

$1 \leq i \leq m, 1 \leq j \leq n, 2 \leq k \leq m+1,1 \leq s \leq 2$

Input objects in membrane 2: check clauses

D1 (check if the clause is satisfied by at least one variable)

$\left[v_{j, i, m+j, 1} \rightarrow v_{j, i, k+1,3} o_{1,4}\right]^{2}, 1 \leq i \leq m, 1 \leq j \leq n, 1 \leq s \leq 2$ 
D2 (mark an object)

$\left[v_{j, i, m+k, s} \rightarrow v_{j, i, k+1, s+2} o_{1,4}\right]^{1}$,

$1 \leq i \leq m, 1 \leq j \leq n, 1 \leq k \leq n, 1 \leq s \leq 2$

D3 (unmark an object)

$$
\begin{aligned}
& {\left[v_{j, i, m+k, s+2} \rightarrow v_{j, i, k, s} o_{3,4}\right]^{3},} \\
& 1 \leq i \leq m, 1 \leq j \leq n, 2 \leq k \leq n+1,1 \leq s \leq 2
\end{aligned}
$$

\section{Control objects in membrane 3}

E1 (count)

$$
\left[z_{k-1} \rightarrow z_{k}\right]^{0}, 1 \leq k \leq N=(10 l+5) n+m(10 l+1)+2
$$

E2 (send time-out object)

$$
\left[z_{N}\right]^{0} \rightarrow[]^{0} Z
$$

\section{Control objects in the skin membrane}

F1 (the first positive result sends the answer)

$$
[S]^{0} \rightarrow[]^{1} \text { yes }
$$

F2 (without the positive result, the time-out sends the negative answer)

$$
[\mathrm{Z}]^{0} \rightarrow[]^{0} \mathrm{no}
$$

Let us now explain how the system works in greater detail. The control objects keep track of the number of cycles completed, whether marking or unmarking takes place, as well as the number of objects already (un)marked. Moreover, the control object is responsible for sending the "right" information to the objects via polarization: in stage 1 , by generating $o_{0,1}$ or $0_{5,1}$ if the variable is true, and $o_{0,2}$ or $o_{5,2}$ if the variable is false; in stage 2, $o_{0,1}$ or $o_{5,1}$ if the clause has already been found, and $o_{0,2}$ or $o_{5,2}$ if the clause is being checked for.

During the first stage, an object $v_{j, i, 1, s}$ is transformed into $v_{j, i, n+1, t}$, where $t=1$ if variable $x_{j}$ satisfies clause $C_{i}$, or $t=2$ if not. The last index changes from $s$ to $t$ happens when the third index is equal to $i$. The control object $d_{1}$ is transformed into $d_{n+1}$. Stage 1 takes at most $(10 l+5) n$ steps (at most $(10 l+3) n$ in the case when $l$ is even).

If some clause is not satisfied, then the computation in the corresponding membrane is "stuck" with polarization 2. Otherwise, during the second stage an object $v_{j, i, n+1, t}$ is transformed into $v_{j, i, n+m+1, t}$, while the control object $d_{n+1}$ becomes $d_{m+n+1}$. Stage 2 takes at most $m(10 l+1)$ steps, plus one extra step to send objects $S$ to skin, if any.

After stage 2 is completed, one copy of $S$, if any, is sent out as yes, changing the polarization of the skin membrane. After this time has passed, 
object $Z$ comes to the skin from region 3. If the polarization of the skin is still $0, Z$ is sent out as no.

The rules of the system in the proof above are also global, so we can again obtain the following

Corollary 2. A uniform family of confluent polarizationless $P$ systems with rules $(a),\left(c_{0}^{\prime}\right),\left(e_{0}\right)$ working in minimally parallel way can solve SAT with membrane labels of six kinds.

\section{Conclusions}

Changing membrane polarization controls what rules can be applied, so the number of polarizations corresponds to the number of states of this control. Moreover, almost the only way the objects of the system may interact is by changing the membrane polarization. Therefore, the number of polarizations is a complexity measure that deserves our attention.

For maximal parallelism it has been proved that two polarizations are sufficient for both universality (with one membrane) and efficiency, while one-polarization systems are still universal (with elementary membrane division and membrane dissolution), but are conjectured not to be efficient.

We have proved that efficient solutions of computationally hard problems by $\mathrm{P}$ systems with active membranes working in a minimally parallel way can be constructed avoiding both cooperative rules and non-elementary membrane division, thus improving results from [7],[8]. For this task, it is enough to have four polarizations, sequential evolution rules changing polarizations, polarizationless elementary membrane division rules and polarizationless rules for sending an object out. The standard evolution and send-out rules, as well as polarizationless elementary membrane division rules, can be used; in this case, six polarizations suffice.

The first construction is "almost" deterministic: the only choices the system can make in each cycle is the order in which the input systems are processed. The second construction exhibits a more asynchronous behaviour of the input objects, which, depending on the chosen degree of parallelism, might speed up the positive answer, but by less than $20 \%{ }^{2}$ In this case,

2 The maximal total number of steps needed is slightly over $10 l(m+n)$; the fastest computation happens if rules $\mathrm{C} 2$ are executed in parallel for all input objects, as well as rules $C 4, D 2, D 3$, saving $l m-1, \operatorname{lm}-1, \ln -1, \ln -1$ steps, respectively.

TRIANGLE 6 • December 2011 
controlling polarizations by evolution is still faster than controlling polarizations by communication.

A number of interesting problems related to minimal parallelism remain open. For instance, is it possible to decrease the number of polarizations/labels? Other computational problems in the minimally-parallel setting can also be studied: for instance, the computational power of P systems with one active membrane working in the minimally parallel way.

\section{References}

1. A. Alhazov, P Systems without Multiplicities of Symbol-Objects, Information Processing Letters 100 (3), 2006, 124-129.

2. A. Alhazov, L. Pan, Gh. Păun, Trading Polarizations for Labels in P Systems with Active Membranes, Acta Informatica 41, 2-3, 2004, 111-144.

3. A. Alhazov, R. Freund, On the Efficiency of P Systems with Active Membranes and Two Polarizations, Membrane Computing, International Workshop, WMC 2004, Milan, 2004, Revised Selected and Invited Papers (G. Mauri, Gh. Păun, M.J. Pérez-Jiménez, G. Rozenberg, A. Salomaa, Eds.), Lecture Notes in Computer Science 3365, Springer, 2005, 146-160, and Fifth Workshop on Membrane Computing (WMC5) (G. Mauri, Gh. Păun, C. Zandron, Eds.), University of Milano-Bicocca, Milan, 2004, 81-94.

4. A. Alhazov, R. Freund, A. Riscos-Núñez, One and Two Polarizations, Membrane Creation and Objects Complexity in P Systems, Seventh International Symposium on Symbolic and Numeric Algorithms for Scientific Computing (SYNASC'05), IEEE Computer Society, 2005, 385-394, and First International Workshop on Theory and Application of P Systems, Timişoara, Romania, 2005 (G. Ciobanu, Gh. Păun, Eds.), 2005, 9-18.

5. A. Alhazov, R. Freund, A. Riscos-Núñez, Membrane Division, Restricted Membrane Creation and Object Complexity in P Systems. International Journal of Computer Mathematics 83 (7), 2006, 529-548.

6. A. Alhazov, R. Freund, Gh. Păun, Computational Completeness of P Systems with Active Membranes and Two Polarizations, Machines, Computations, and Universality, International Conference, MCU 2004, Saint Petersburg, 2004, Revised Selected Papers (M. Margenstern, Ed.), Lecture Notes in Computer Science 3354, Springer, 2005, 82-92.

7. G. Ciobanu, Gh. Păun, L. Pan, M.J. Pérez-Jiménez, P Systems with Minimal Parallelism, Theoretical Computer Science 378 (1), 2007, 117-130.

Their total is $2 l(m+n)-4$, which is less than (but assymptotically equal to) $1 / 5$ of the worst time. 
8. T.-O. Ishdorj, Minimal Parallelism for Polarizationless P Systems, 12th International Meeting on DNA Computing (DNA12), Seoul, 2006, Revised Selected Papers (C. Mao, T. Yokomori, Eds.), Lecture Notes in Computer Science 4287, 2006, 17-32.

9. Gh. Păun, P Systems with Active Membranes: Attacking NP-Complete Problems, Journal of Automata, Languages and Combinatorics 6, 1, 2001, 75-90.

10. Gh. Păun, Membrane Computing. An Introduction, Springer-Verlag, Berlin (Natural Computing Series), 2002.

11. Gh. Păun, Y. Suzuki, H. Tanaka, T. Yokomori, On the Power of Membrane Division in P systems, Theoretical Computer Science 324, 1, 2004, 61-85.

12. M.J. Pérez-Jiménez, A. Romero-Jiménez, F. Sancho-Caparrini, Complexity Classes in Cellular Computing with Membranes, Natural Computing 2, 3, 2003, 265-285.

13. P Systems Webpage, http://ppage.psystems .eu/ 